\title{
Effects of fimbria-fornix lesions on rats' use of intramaze stimuli in the water maze
}

\author{
TOSHIRO SAKAMOTO and HIROSHIGE OKAICHI \\ Doshisha University, Kyoto, Japan
}

\begin{abstract}
In order to examine the role of the hippocampus in spatial learning, fimbria-fornix-lesioned (FF) rats and control rats were trained in a water maze enclosed in black curtains. In the condition with stimuli, two objects of different appearance were hung on the water surface as intramaze stimuli. The hidden goal and two objects formed a triangle, and their spatial relationship was kept constant throughout the training. For the condition without stimuli, the two stimuli were removed. In the condition with stimuli, the control group solved the task faster than the FF group. Analysis of navigational strategy indicated that the control group used both the place strategy and the response strategy, whereas the FF group used only the response strategy. In the condition without stimuli, the performance of both groups was similar, with both groups solving the task using only the response strategy. These results suggest that the hippocampus plays an important role in utilization of the spatial relationships among intramaze stimuli.
\end{abstract}

Many researchers have reported that the hippocampus plays an important role in processing spatial information (Jarrard, 1993; O'Keefe, 1991; O'Keefe \& Nadel, 1978; Poucet, 1993). It has been shown that rats cannot solve the place learning tasks in various mazes when the hippocampus or fimbria-fornix is lesioned (e.g., the T maze, Okaichi, 1987; the Y maze, Aggleton, Hunt, \& Rawlins, 1986; the radial arm maze, Jarrard, 1983; Jarrard, Okaichi, Steward, \& Goldschmidt, 1984; Nadel \& MacDonald, 1980; Okaichi \& Oshima, 1990; Pico, Gerbrandt, Pondel, \& Ivy, 1985; the water maze, Morris, Garrud, Rawlins, \& O'Keefe, 1982; Morris, Hagan, \& Rawlins, 1986). However, hippocampal- or fimbria-fornix-lesioned (FF) rats, like normal rats, can solve cue learning tasks (Aggleton et al., 1986; Jarrard, 1983; Morris et al., 1982; Morris et al., 1986; Nadel \& MacDonald, 1980; Okaichi, 1987; O'Keefe \& Conway, 1980).

In the place task - for example, the place learning version of the water maze task-rats must learn to locate a hidden platform by utilizing the spatial relationships among various extramaze stimuli, because there are no cues in the maze that directly indicate a goal. In contrast, in the cue learning version of the water maze task, rats need learn only to reach a visible platform because there are direct cues in the maze. The visual stimuli in the place tasks have usually been extramaze stimuli--that is, distal stimuli arranged in a room. In the cue tasks, the available visual cues have usually been intramaze stimuli placed in a maze - that is, proximal stimuli. Therefore, it is thought

This research was supported by the Research Fellow of the Japan Society for the Promotion of Science. The authors are grateful to Dr. Shizuhiko Nishisato (Toronto University) and two referees for their useful comments. Correspondence should be addressed to T. Sakamoto, Department of Psychology, Doshisha University, Kyoto 602, Japan (e-mail: tsakamot@mail.doshisha.ac.jp). that the behavioral impairment of hippocampal rats in place tasks might simply manifest as inability to use the distal visual stimuli.

O'Keefe and Conway (1980) showed that FF rats were able to use extramaze stimuli if they served as cues. Using a cross maze, they trained rats on two versions of the stimulus-controlled task. In the distributed-stimulus version, visual stimuli were dispersed around a room in such a way that no cues were set directly behind the goal arm; in the clustered-stimulus version, the same visual stimuli were all placed directly behind the goal arm. Whereas normal rats could solve both versions, FF rats could solve only the clustered-stimuli version. This indicates that FF lesions impair the use of the spatial relationships among extramaze stimuli and do not impair the use of extramaze stimuli in themselves.

In the place learning tasks of the studies mentioned, only extramaze stimuli were used and controlled. Hippocampal rats could not solve the tasks by using the spatial relationships among them. According to the spatial mapping hypothesis (O'Keefe, 1991; O'Keefe \& Nadel, 1978), it is inferred that hippocampal rats fail to utilize an array of intramaze stimuli as well. However, few researchers have studied whether hippocampal rats can use the spatial relationships among intramaze stimuli.

Collet, Cartwright, and Smith (1986) showed that normal gerbils can utilize the spatial relationships among intramaze stimuli. In their experiments, normal gerbils could locate buried food in reference to an array of three objects in a circular open field. Collet et al. used only normal animals and therefore did not consider the role of the hippocampus in the use of intramaze stimuli. Save, Poucet, Foreman, and Buhot (1992) reported that hippocampal rats could not recognize a spatial change of familiar intramaze stimuli. In their research, hippocampal rats were compared with control rats for their exploration of five 
objects in a circular open field, habituation of object investigation, and response to spatial and nonspatial change. After both groups displayed habituation and decreased exploration activity toward the five objects, some of the objects were displaced in a test session. Control rats selectively reexplored displaced objects, but hippocampal rats did not react to the spatial change. When one of five objects was replaced by a new one in an additional test session, both groups explored the new one. These results indicate that the hippocampus is involved in recognizing spatial information of proximal stimuli in an open field.

Gothard, Skaggs, Moore, and McNaughton (1996) examined the behavioral correlation of rat hippocampal CAl cell activity in a spatial navigation task in which two landmarks predicted the location of food. The landmarks were maintained at a constant distance from each other but were moved from trial to trial within an arena surrounded by static background cues. The discharge characteristics of multiple, simultaneously recorded cells were examined with respect to the landmarks (intramaze stimuli) and static background cues (extramaze stimuli). The outcome was that there were four times as many cells with location-specific firing (place cells) as cells that fired in the goal or landmarks, regardless of their location in the arena. Cressant, Muller, \& Poucet (1997) showed under similar circumstances that two objects located in an arena are not sufficient for stimulus control to be exerted over place cell activity.

The results of Gothard et al. (1996) and Cressant et al. (1997) suggest that the hippocampus is not involved to the same extent in the processing of proximal versus distal information. Accordingly, it is meaningful to examine--not only through recording studies but also through lesion studies - whether hippocampal rats can use the spatial relationships among intramaze stimuli.

We used the water maze and designed a place learning task that rats could solve using an array of intramaze stimuli. The Morris water maze task was designed to eliminate the problem of intramaze stimuli. Therefore, many studies have focused on extramaze stimuli. In our experiment, the water maze was enclosed by curtains, so extramaze stimuli were unavailable. Under the condition with stimuli, the hidden goal and two stimuli of different appearance were placed in a pool, forming a triangle. Therefore, rats had to locate the platform by recognizing an array of these two stimuli. However, the two stimuli were removed for the condition without stimuli. The rats' performances under these two conditions were compared in order to examine the use of intramaze stimuli.

The purpose of the present experiment was to observe escape training in two conditions (i.e., with stimuli and without stimuli) for fimbria-fornix-lesioned (FF) rats and control rats and to examine the effect of the FF lesions on rats' ability to use the spatial relationships among intramaze stimuli in solving the water maze task. Moreover, we recorded all navigational paths throughout the training and analyzed control rats' and FF rats' strategies in solving the two kinds of tasks.

\section{METHOD}

\section{Subjects}

The subjects were 40 naive male albino rats of the Wistar strain. The rats were assigned to two conditions, the condition with stimuli $(n=20)$ and the condition without stimuli $(n=20)$. For each condition, the rats were assigned to an FF-lesioned $(n=10)$ or a control $(n=10)$ group. Their mean weight was $275.1 \mathrm{~g}(S D=12.1 \mathrm{~g})$ at the beginning of the experiment. Throughout the experiment, all animals were housed individually in standard wire-mesh cages and given free access to food and water. Lighting was alternated in a 12:12-h light:dark cycle starting at 7:00 A.M. in a temperature- and humidity- controlled room.

\section{Apparatus}

The swimming pool (diameter $142 \mathrm{~cm}$, height $80 \mathrm{~cm}$ ) was a circular black polyester tank located in the center of a dimly lit room and enclosed in black curtains. The pool was filled to a depth of $59 \mathrm{~cm}$ with water $\left(24^{\circ} \pm 1^{\circ}\right)$, which was rendered opaque by the addition of $100 \mathrm{ml}$ of black ink. Illumination intensity at the water surface was $20 \mathrm{~lx}$.

An escape platform, a black column measuring $11 \mathrm{~cm}$ in diameter and $57.5 \mathrm{~cm}$ in height, was hidden in the pool. In the condition with stimuli, two electric bulbs served as intramaze stimuli, a blue light ( $7 \mathrm{~W}$, Stimulus A) and a yellow flicker light (10 W, Stimulus B). These were hung on the water surface from two timber beams (each $1 \mathrm{~cm} \times 1 \mathrm{~cm}, 150 \mathrm{~cm}$ long) forming a cross over the pool.

All navigational paths were recorded on videotape with a camera set below the ceiling and were analyzed with a tracking system (Biomedica, Osaka) run on a personal computer (NEC, PC-9801VX).

\section{Surgery and Histology}

All FF rats $(n=20)$ were deeply anesthetized with $50 \mathrm{mg} / \mathrm{kg}$ of sodium pentobarbital, i.p., and then treated with $0.1 \mathrm{cc}$ atropin sulfate, i.p. The FF lesions were produced by a Radionics RFG-4. Four electrode sites were used $(\mathrm{AP}=-2.1 \mathrm{~mm}, \mathrm{ML}= \pm 1.2$ and $\pm 2.7 \mathrm{~mm}$ from the bregma, $\mathrm{DV}=-3.8 \mathrm{~mm}$ from the surface of the dura). The temperature at the tip (diameter $0.7 \mathrm{~mm}$, height $1 \mathrm{~mm}$ ) of the electrode was maintained at $55^{\circ}$ for $60 \mathrm{sec}$.

At the conclusion of behavioral testing, each animal was deeply anesthetized with $20 \%$ chloral hydrate solution and perfused intracardially with $10 \%$ formalin. The brains were removed, sectioned $40 \mu \mathrm{m}$ thick on a freezing microtome, and stained with cresyl violet.

\section{Procedure}

The rats were handled for 5 min each for 6 days before and after surgery. One week after the surgery, all animals were subjected to escape training.

Condition with stimuli. The platform was $35 \mathrm{~cm}$ away from a wall of the pool. Stimulus A, Stimulus B, and the platform formed a triangle in the pool. The platform was $35 \mathrm{~cm}$ away from Stimulus A (or Stimulus B) and $65 \mathrm{~cm}$ away from Stimulus B (or Stimulus A). Stimulus A was $80 \mathrm{~cm}$ away from Stimuli B. This triangle was kept constant in the pool throughout the training. Figure 1 shows the water maze containing these stimuli, the platform, and the black curtains.

In each training trial, the rat was gently placed in the water facing the wall at the designated start position. A maximum of $60 \mathrm{sec}$ was allowed for each trial, and any rat that did not locate the escape platform within this time was taken out of the water and placed onto the platform by the experimenter. After finding the platform or being placed on the platform, the rat stayed there for about $20 \mathrm{sec}$ and was then returned to a holding cage.

Four equally spaced points, arbitrarily designated by compass points $(\mathrm{N}, \mathrm{S}, \mathrm{E}, \mathrm{W})$, were used as starting positions around the edge of the pool. The platform was shifted to the center position of one of four quadrants (NE, NW, SE, SW) for every trial, while the spa- 

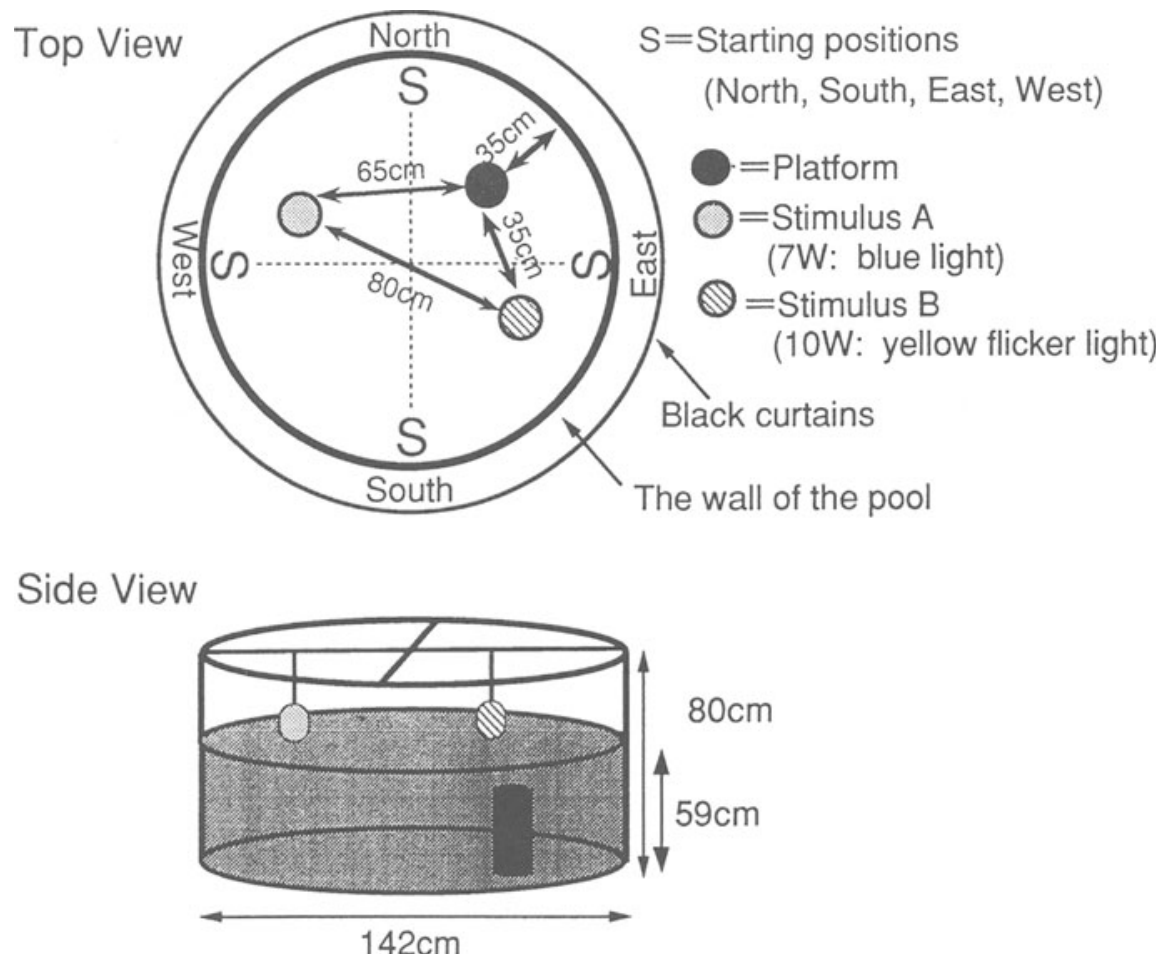

Figure 1. Illustration of the water maze and black curtains in the condition with stimuli. The locations of Stimuli $A$ and $B$ were counterbalanced for every rat in the condition with stimuli. The two stimuli and timber cross were removed for the condition without stimuli.

tial relationship between the platform and the two stimuli in the pool was kept constant. Four different combinations of platform positions and starting positions were randomly assigned to four successive trials. Each rat was given 2 daily trials for 20 days for a total of 40 trials.

After the training, each rat was given two kinds of probe tests for four test trials each. In Probe Test A, the two stimuli were removed. In Probe Test B, although the two stimuli were removed, the escape platform, painted white, was positioned $2 \mathrm{~cm}$ above the water surface. The position of the platform was shifted for every trial in both Probe Test A and Probe Test B.

Condition without stimuli. Each rat was given the same escape training as in the condition with stimuli, except that the two stimuli and the timber cross were removed before the training, and the two probe tests were not practiced. During the training, each rat was given two daily trials for 20 days for a total of 40 trials.

\section{RESULTS}

\section{Histology}

Figure 2 is a drawing of representative FF lesions. In each lesioned rat, the fimbria-fornix and anterior parts of the dorsal hippocampus were severed bilaterally. In the maximum extent of lesions (indicated by the light gray in Figure 2), portions of the lateral septum and posterior parts of the dorsal hippocampus were damaged. Portions of the corpus callosum and stria terminals were also damaged. In the minimum extent of lesions (indicated by the dark gray in Figure 2), portions of the lateral septum and the corpus callosum were damaged, but the stria terminals were left intact. FF rats had some damage to the parts of the neocortex through which the electrode had been inserted. There was no evidence of any relation between the extent of lesion and performance.

\section{Behavior}

Swimming latencies of the FF group and the control group. For the condition with stimuli, logarithmic values of the mean latencies of the FF group and the control group for each block of four trials are shown in Figure $3 \mathrm{~A}$. The mean latencies of both groups decreased similarly at the beginning of the training, but the mean latencies of the control group decreased to a greater extent than those of the FF group in the latter parts of the training.

Statistical analysis of the data was conducted using a factorial-design analysis of variance (ANOVA) with group (FF, control) as a between-subjects factor and block (1 st10 th block) as a within-subject factor. Analysis of logarithmic values of the latencies indicated that the main effects of group $[F(1,18)=8.13, p<.01]$ and block $[F(9,162)=18.24, p<.01]$ and the group $\times$ block interaction $[F(9,162)=2.20, p<.05]$ were significant. Tests of simple main effects revealed significant differences 


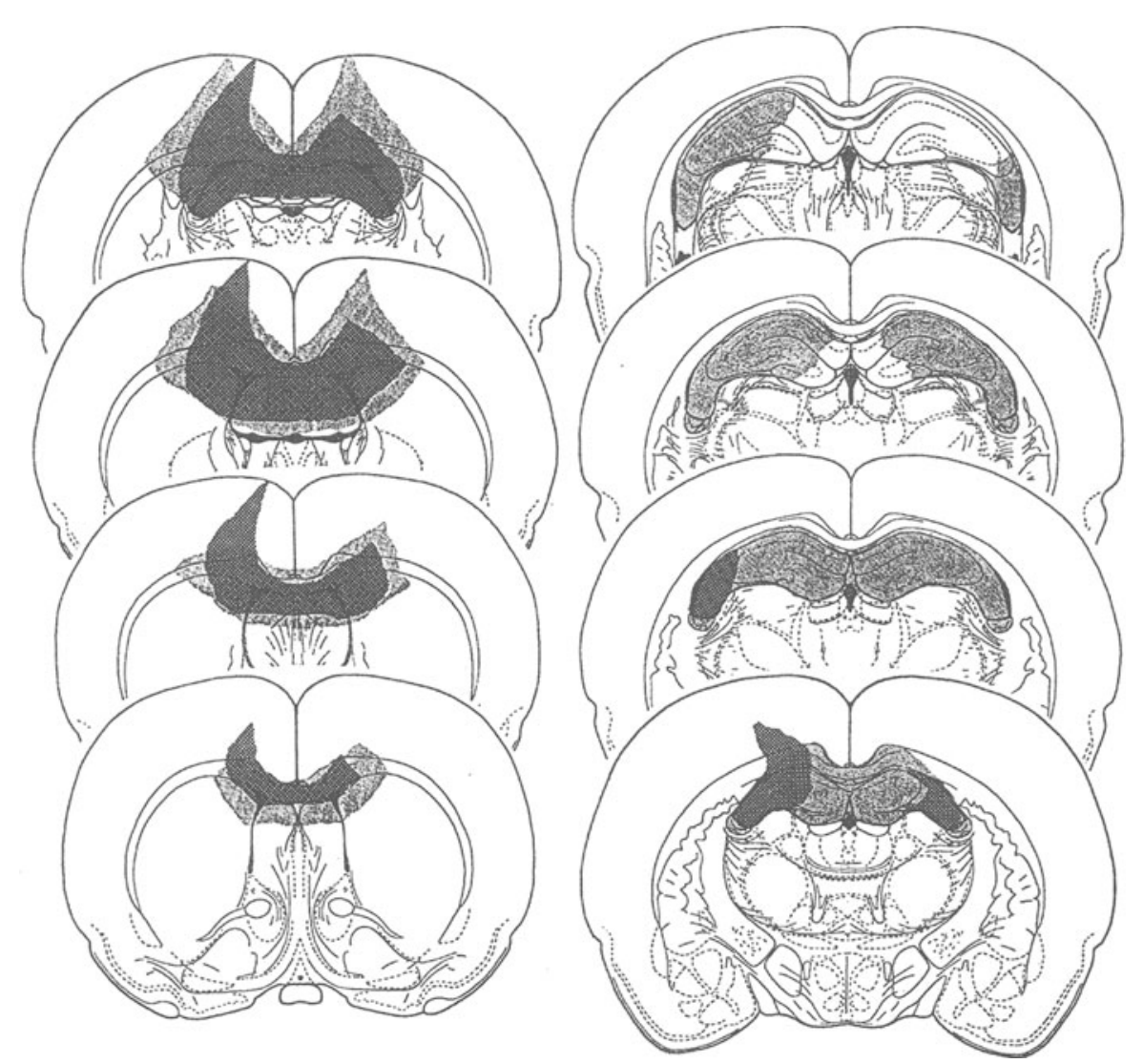

Figure 2. Examples of fimbria-fornix lesions (dark gray indicates the minimum extent of lesions; light gray indicates the maximum extent of lesions).

between the FF group and the control group on the 5th block $(p<.05)$, the 7 th block $(p<.01)$, the 8 th block $(p<$ $.01)$, the 9th block $(p<.01)$, and the 10th block $(p<.01)$.

Logarithmic values of the mean latencies for each block of four trials in the condition without stimuli are shown in Figure 3B. The mean latencies of both groups decreased similarly throughout the training. An ANOVA was conducted for group $(\mathrm{FF}$, control) $\times$ block (1st-10th). Analysis of logarithmic values of the latencies indicated that the main effect of block $[F(9,162)=8.62, p<.01]$ was significant, but the main effect of groups and the group $X$ block interaction were not significant.

Classification and analysis of rats' strategies. We used the measure ASRS (the amount of successively rotational swimming) to examine navigational strategies. Figure 4 shows examples of ASRS measurement. We calculated each rat's ASRS for the last four trials in which swimming latency was within $25 \mathrm{sec}$ (when swimming latency was over $25 \mathrm{sec}$ throughout the training, the rat's ASRS was 0). We then classified the rats into high-ASRS (more than 2 per trial) and low-ASRS (less than 2 per trial) groups.

The ASRS shows whether rats used the response strategy in solving the task. The high-ASRS rats solved two kinds of tasks by swimming around a certain distance from the wall of the pool, so it is assumed that they used the response strategy. The low-ASRS rats did not use the response strategy.

In the condition with stimuli, the control group was divided into two groups -5 rats in the high-ASRS group (CHl) and 5 rats in the low-ASRS group (CLl). The FF group was also divided, with 7 rats in the high-ASRS group (FH1) and 3 in the low-ASRS group (FL1). Figure 5 shows the navigational paths of the representative rats in the $\mathrm{CH} 1$, the $\mathrm{FH} 1$, and the CL1 groups. Rats in both the $\mathrm{CH} 1$ and the $\mathrm{FH} 1$ groups solved the task using the response strategy, as noted. Although both the CLl and the FL1 rats belonged to the low-ASRS group, they used completely different strategies. The rats in the CLl group could reach the platform directly, which means that they used the place strategy. The animals in the FL1 did not use any strategies in solving this task. They swam along the wall of the pool or swam randomly.

Logarithmic values of the mean swimming latencies of the $\mathrm{CH} 1$ and the CL1 rats in the training period and two probe tests are shown in Figure 6A. An ANOVA was conducted for group (CH1, CL1) $\times$ block (1st-10th). Analysis of logarithmic values of the mean latencies in- 


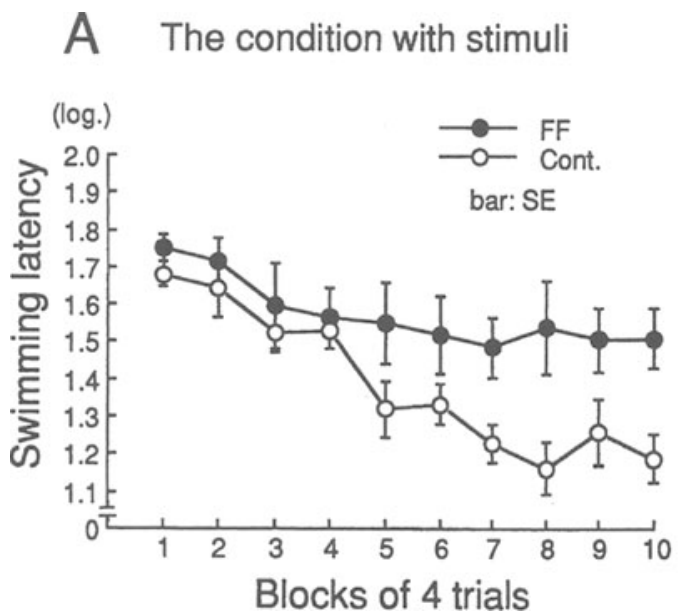

\section{B The condition without stimuli}

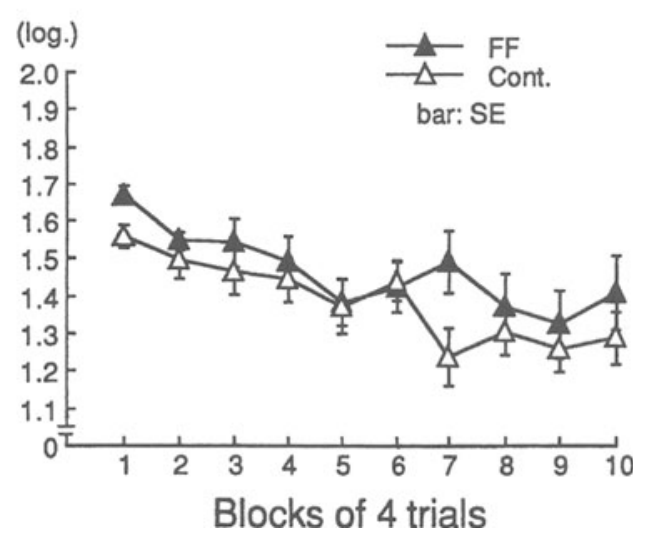

Figure 3. Swimming latencies of the fimbria-fornix-lesioned (FF) group and the control group.

dicated that the main effect of block was significant $[F(9,72)=13.89, p<.01]$, and that the main effect of group and the group $\times$ block interaction were not significant. The results of the ANOVA for group $(\mathrm{CH}, \mathrm{CLl}) \times$ test (10th block, Probe Test A, Probe Test B) indicated that the main effect of group $[F(1,8)=5.83, p<.05]$ and the group $\times$ test interaction $[F(2,16)=4.58, p<.05]$ were significant. Tests of simple main effects revealed significant differences between the $\mathrm{CH} 1$ and the $\mathrm{CL} 1$ rats in Probe Tests A and B.

Logarithmic values of the mean swimming latencies of the $\mathrm{FH} 1$ and the $\mathrm{FL} 1$ rats in the training period and the two probe tests are shown in Figure 6B. An ANOVA was conducted for group $(\mathrm{FH} 1, \mathrm{FL} 1) \times$ block (1st-10th). Analysis of logarithmic values of the latencies indicated that the main effect of group was almost significant $[F(1,8)=$

\section{(S) = Starting position $=$ Platform}
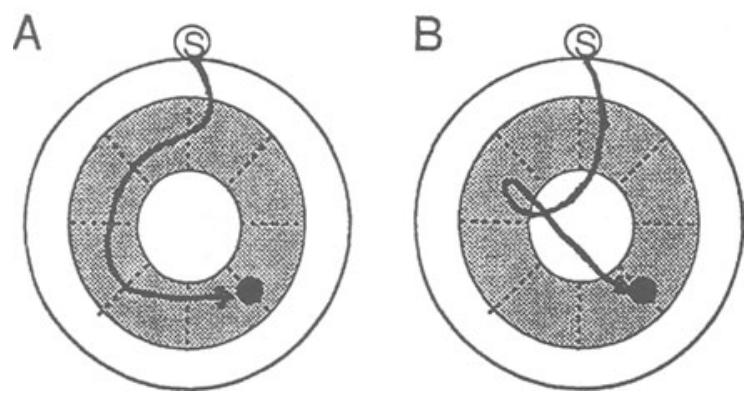

Figure 4. Examples of measurement of the amount of successively rotational swimming (ASRS). The ASRS refers to the greatest number of times that a rat crosses the border of a neighboring zone. There are eight zones (shaded gray; inside radius $20 \mathrm{~cm}$, outside radius $50 \mathrm{~cm}$. The platform is $35 \mathrm{~cm}$ from the center and wall of the pool). The ASRS is 5 in Figure $4 A$ and 0 in Figure $4 B$.
$4.81, p<.06]$, whereas the main effect of block $[F(9,72)=$ $3.31, p<.01]$ and the group $\times$ block interaction $[F(9,72)=$ $2.14, p<.05]$ were significant. Tests of simple main effects revealed significant differences between the groups in the 6th, 7th, 8th, 9th, and 10th blocks (all $p s<.05$ ). The simple main effect of block for the FH1 rats was significant $(p<.05)$. Results of the ANOVA for group (FH1, FL1) $\times$ test (10th block, Probe Test A, Probe Test B) indicated that the main effect of group $[F(1,8)=12.95$, $p<.01]$ was significant and that the group $\times$ test interaction $[F(2,16)=2.78, p<.10]$ was nearly significant.

In the condition without stimuli, the control group was divided into two groups, with 6 rats in the high-ASRS group ( $\mathrm{CH} 2$ ) and 4 rats in the low-ASRS group (CL2). The FF group was also divided, with 7 rats in the high-ASRS group (FH2) and 3 rats in the low-ASRS group (FL2). Figure 7 shows the navigational paths of representative rats in the $\mathrm{CH} 2$ and the $\mathrm{FH} 2$ groups. Rats in both the $\mathrm{CH} 2$ and the $\mathrm{FH} 2$ groups used the response strategy, as did the $\mathrm{CH} 1$ and the $\mathrm{FH} 1$ rats in the condition with stimuli. Like the FL1 rats, animals in both the CL2 and the FL2 groups did not use strategies; they swam along the wall of the pool or swam randomly.

Logarithmic values of the mean swimming latencies of the $\mathrm{CH} 2$ and the CL2 rats in the training period are shown in Figure 8A. An ANOVA was conducted for group (CH2, CL2) $\times$ block (1 st-10th). Analysis of logarithmic values of the latencies indicated that the main effects of group $[F(1,8)=11.14, p<.01]$ and block $[F(9,72)=3.44, p<.01]$ were significant and that the group $\times$ block interaction $[F(9,72)=1.76, p<.10]$ was nearly significant.

Logarithmic values of the mean swimming latencies of the $\mathrm{FH} 2$ and the FL2 rats in the training period are shown in Figure 8B. An ANOVA was conducted for group (FH2, FL2) $\times$ block (1st-10th). Analysis of logarithmic values of the latencies indicated that the main effects of group $[F(1,8)=12.48, p<.01]$ and block 
A The $\mathrm{CH} 1$ RAT 04
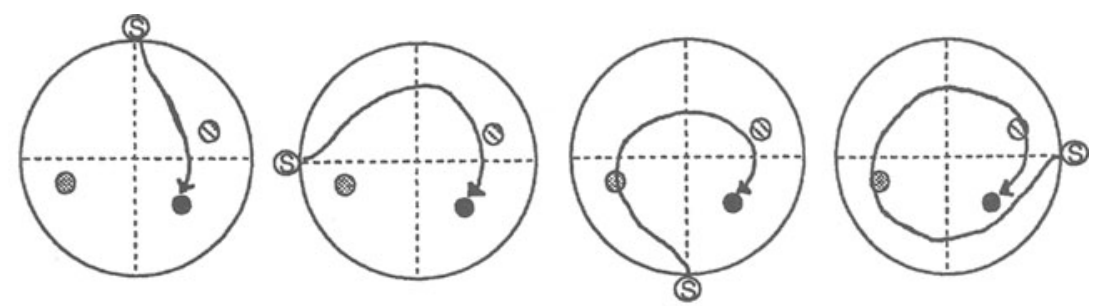

The CL1 RAT 18
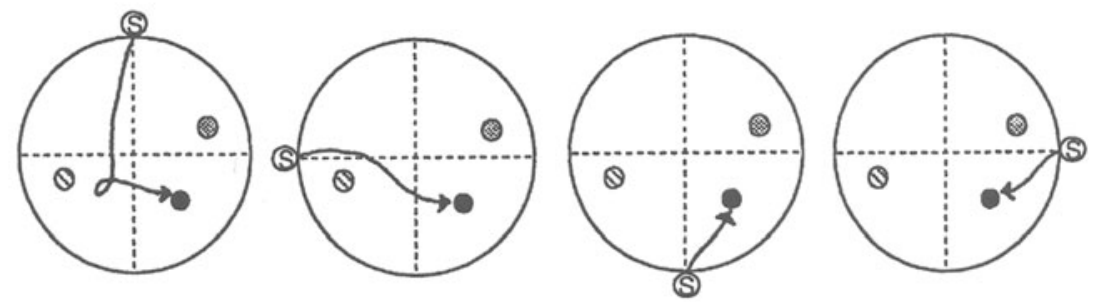

The FH1 RAT 11
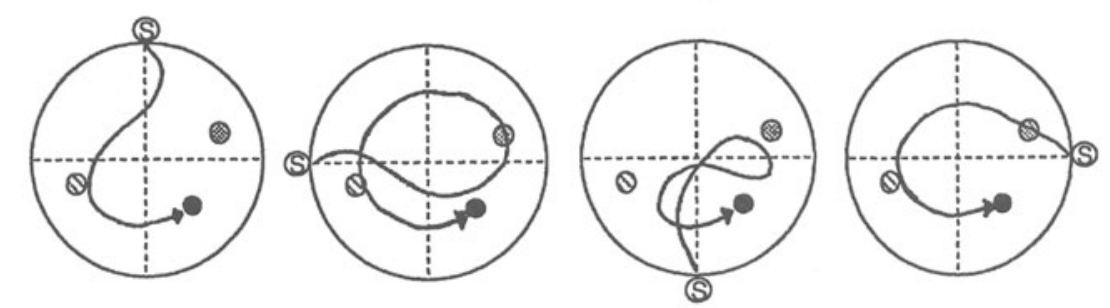

\section{S $=$ Starting positions \\ =Platform \\ - $=$ Stimulus $A$ \\ $\theta=$ Stimulus B}

Figure 5. Typical examples of paths of the CH1 $(n=5)$, CL1 $(n=5)$, and FH1 $(n=7)$ groups in the condition with stimuli. Navigational paths in the final four trials were completed within $25 \mathrm{sec}$, showing different relations between the starting positions and the platform position. CH1, high-ASRS control, with stimuli; CL1, low-ASRS control, with stimuli; FH1, high-ASRS FF, with stimuli; ASRS, amount of successively rotational swimming; FF, fimbria-fornix-lesioned.

$[F(9,72)=3.55, p<.01]$ were significant and that the group $\times$ block interaction was not significant.

\section{DISCUSSION}

\section{Swimming Latencies of the FF Group and the Control Group}

The findings for swimming latencies in both conditions suggest that the hippocampus is involved in utilization of the spatial relationships among intramaze stimuli. These results are consistent with results reported by Save et al. (1992), who demonstrated that the hippocampal-lesioned rats could not recognize the spatial relationships among intramaze stimuli.

\section{Classification and Analysis of Rats' Strategies}

In the condition with stimuli, some control rats ( $\mathrm{CL} 1$ rats, $n=5$ ) could reach the platform directly by utilizing an array of the two stimuli; that is, they used the place strategy. The others ( $\mathrm{CH} 1$ rats, $n=5$ ) solved the task by swimming around the pool at a certain distance from the wall, that is, means they used the response strategy.

The CL1 rats located the platform more slowly in Probe Tests $\mathrm{A}$ and $\mathrm{B}$ than in the 10th training block, whereas the $\mathrm{CH} 1$ rats located the platform faster in Probe Tests $\mathrm{A}$ and $B$ than in the 10th training block. The results of these tests show that the two stimuli were important for the rats that used the place strategy to solve the task, but not for the rats that used the response strategy. Half of the control rats could solve the task with the response strategy, but not with the place strategy. It is generally thought that rats' cognitive load in using the place strategy is greater than that in using the response strategy to solve this task. Furthermore, it has been reported that normal rats can use a number of behavioral strategies simultaneously in solving the water maze task (Whishaw \& Mittlemam, 1986). In view of these matters, the control rats in our experiment may have used the response strategy for practical purposes because it is easier, but may also have been able to use the place strategy.

In the FF group, the rats with the response strategy (FH1 rats, $n=7$ ) learned to locate the platform, and the rats without strategies (FLl rats, $n=3$ ) did not locate the platform throughout the training. The FH1 rats located the visible platform (in Probe Test B) faster than the hidden platform (in the 10th training block and Probe Test A). These results show that FF rats can use the response strategy and the cue strategy. 
A Control rats ( $\mathrm{CH} 1$ and $\mathrm{CL} 1)$

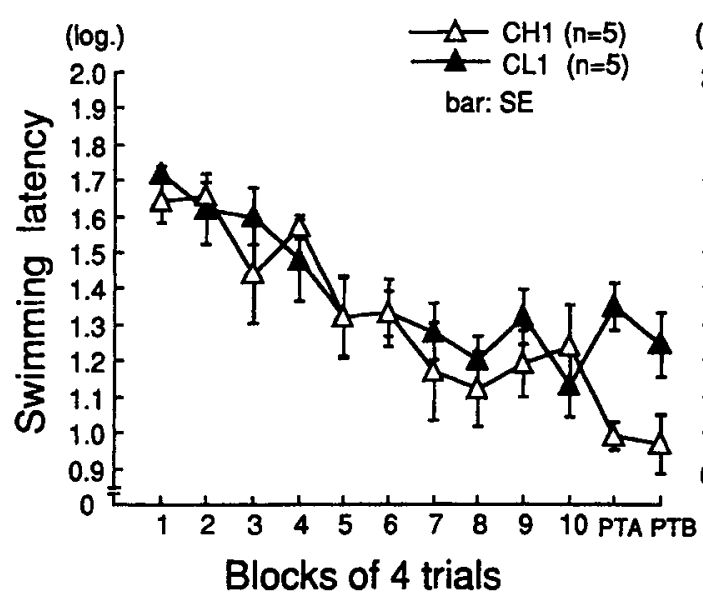

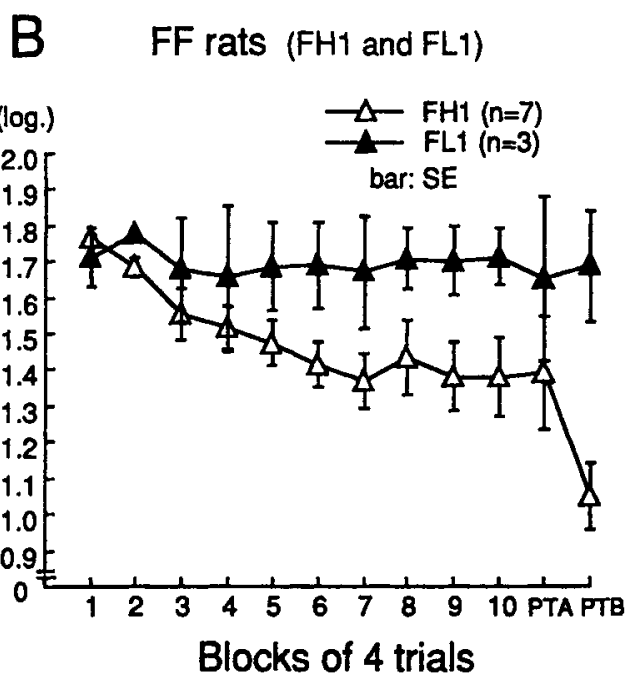

Figure 6. Swimming latencies of each navigational strategy group in the condition with stimuli. PTA, Probe Test $A$, in which the two stimuli were removed and the platform was hidden below the water surface; $P$ TB, Probe Test $B$, in which the two stimuli were removed, but the platform was visible above the water surface.
The $\mathrm{CH} 1$ rats could reach the platform faster than the FHI rats, although the $\mathrm{CHI}$ and the $\mathrm{FHl}$ rats used the same response strategy. When rats use the response strategy in this task, they need to recognize a certain distance from the wall of the pool to the platform. However, it has been reported that FF rats cannot not recognize distance information as accurately as control rats can (Sakamoto \& Okaichi, 1997). Accordingly, it seems that FF rats were less efficient than control rats in using the response strategy. This also suggests that $\mathrm{FH} 1$ rat performance may have improved when the task became easier. Certainly, only the performance of the FHl rats improved in Probe Test $B$, in which a white platform emerged from the surface of the water. The FHl rats learned to escape via the white platform in a few trials because they used the response strategy and had a good chance of crossing the platform. The $\mathrm{CH} 1$ rats did not improve because they accurately used the response strategy and sufficiently learned this task and Probe Test A. The CL1 rats were confused in solving Probe Tests A and B due to the removal of the

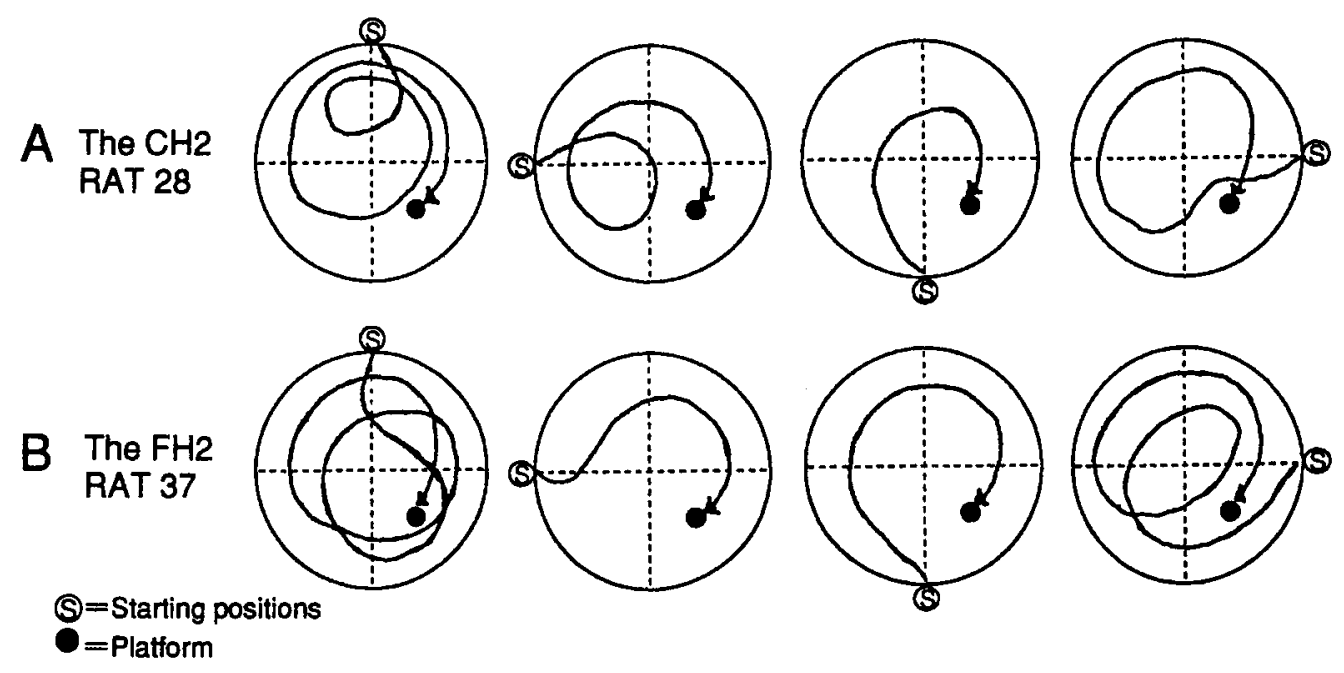

Figure 7. Typical examples of paths of the $\mathrm{CH} 2$ and $\mathrm{FH} 2$ groups in the condition without stimuli. Navigational paths in the final four trials were completed within $25 \mathrm{sec}$, showing different relations between the starting positions and the platform position. CH2, high-ASRS control, without stimuli; FH2, high ASRS FF, without stimuli. ASRS, amount of successively rotational swimming; FF, fimbria-fornix-lesioned. 

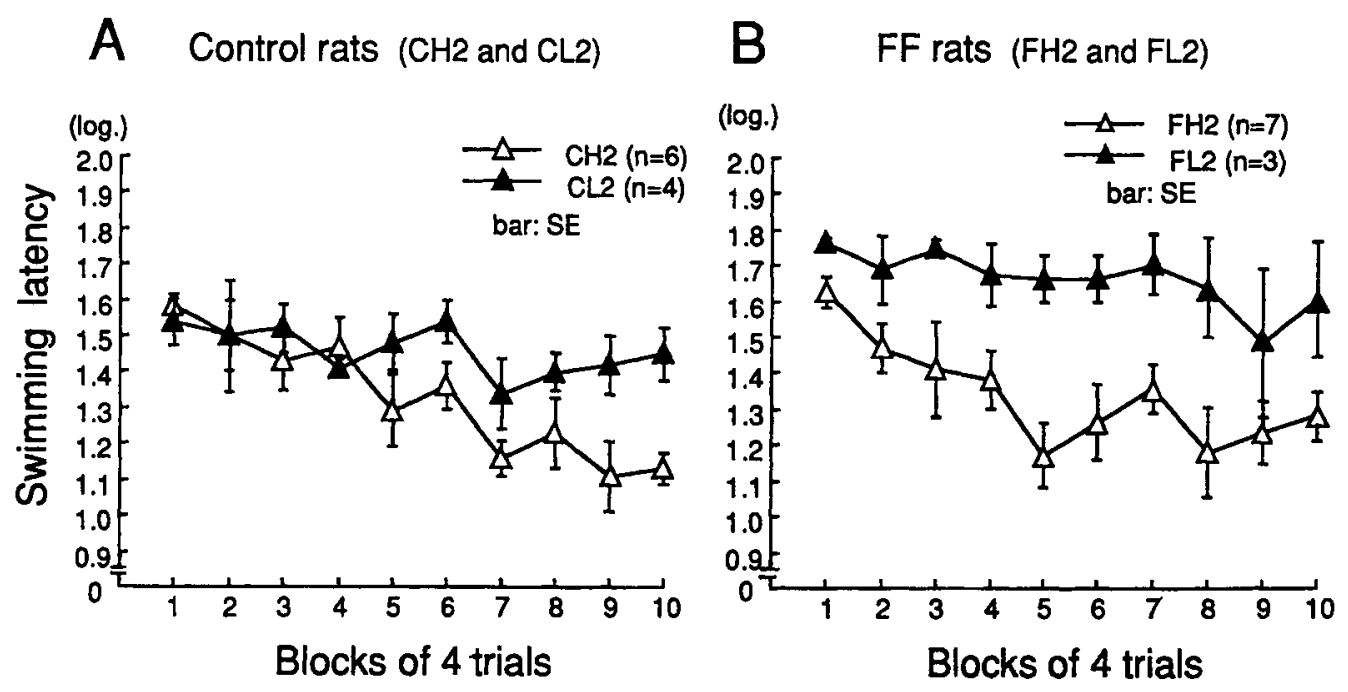

Figure 8. Swimming latencies of each navigational strategy group in the condition without stimuli.

two stimuli (which had been used to solve the task). The FL1 rats went on swimming randomly or along the wall of the pool in Probe Tests A and B.

In the condition without stimuli, some control rats $(\mathrm{CH} 2, n=6)$ and some $\mathrm{FF}$ rats (FH2, $n=7)$ used the response strategy, thereby learning to locate the platform in the condition without stimuli. However, it seems that the $\mathrm{CH} 2$ rats could solve the task a little faster than the $\mathrm{FH} 2$ rats later in the training. The remaining rats in the control group (CL2, $n=4$ ) and in the FF group (FL2, $n=3$ ) did not use any strategies in solving the task and did not learn any strategies throughout the training. These results reveal that rats in both groups could use the response strategy either with or without stimuli.

The most important outcome of this study is that FF rats were not able to navigate toward a goal by using the spatial relationships among intramaze stimuli, whereas control rats were. This indicates that the hippocampus plays an important role in utilization of intramaze stimuli. Many previous studies have shown that hippocampallesioned rats cannot use an array of extramaze stimuli (e.g., Morris et al., 1982; Packard \& McGaugh, 1992). The present findings together with those of previous studies suggest that the hippocampus is generally involved in recognizing the spatial relationships among available stimuli, whether proximal or distal. Moreover, the outcome in lesion studies does not contradict the findings of electrophysiological studies (Cressant et al., 1997; Gothard et al., 1996).

In our experiment, FF rats could use the response strategy and the cue strategy. These results reveal that the hippocampus is scarcely involved in using the response strategy or the cue strategy, consistent with the results in previous water maze studies (cue strategy, Morris et al., 1982; Morris et al., 1986; Packard \& McGaugh, 1992; response strategy, DiMattia \& Kesner, 1988).
In summary, FF rats cannot solve the place learning task by utilizing an array of intramaze stimuli; that is, the hippocampus generally plays an important role in processing the spatial relationships among available stimuli, whether proximal or distal.

\section{REFERENCES}

Aggleton, J. P., Hunt, P. R., \& Rawlins, J. N. P. (1986). The effects of hippocampal lesions upon spatial and nonspatial tests of working memory. Behavioural Brain Research, 19, 133-146.

Collet, T. S., Cartwright, B. A., \& Smith, B. A. (1986). Landmark learning and visuo-spatial memories in gerbil. Journal of Comparative Physiology A, 158, 835-851.

Cressant, A., Muller, R. U., \& Poucet, B. (1997). Failure of centrally placed objects to control the firing fields of hippocampal place cells. Journal of Neuroscience, 17, 2531-2542.

Dimattia, B. V., \& Kesner, R. P. (1988). Role of the posterior parietal association cortex in the processing of spatial event information. Behavioral Neuroscience, 102, 397-403.

Gothard, K. M., SkagGs, W. E., Moore, K. M., \& McNaughton, B. L. (1996). Binding of hippocampal CAl neural activity to multiple reference frames in a landmark-based navigation task. Journal of Neuroscience, 15, 823-835.

JARRARD, L. E. (1983). Selective hippocampal lesions and behavior: Effects of kainic acid lesions on performance of place and cue tasks. Behavioral Neuroscience, 97, 873-889.

JARRARD, L. E. (1993). On the role of the hippocampus in learning and memory in the rat. Behavioral \& Neural Biology, 60, 9-26.

Jarrard, L. E., Okaichi, H., Steward, O., \& Goldschmidt, R. B. (1984). On the role of hippocampal connections in the performance of place and cue tasks: Comparisons with damage to hippocampus. Behavioral Neuroscience, 98, 946-954.

Morris, R. G. M., Garrud, P., Rawlins, J. N. P., \& O' Keefe, J. (1982). Place navigation impaired in rats with hippocampal lesions. Nature, 297, 681-683.

Morris, R. G. M., HaGan, J. J., \& Rawlins, J. N. P. (1986). Allocentric spatial learning by hippocampectomized rats: A further test of the "spatial mapping" and "working memory" theories of hippocampal function. Quarterly Journal of Experimental Psychology, 38B, 365-395.

NADEL, L. , \& MACDonald, L. (1980). Hippocampus: Cognitive map or working memory? Behavioral \& Neural Biology, 29, 405-409. 
OKAICHI, H. (1987). Performance and dominant strategies on place and cue tasks following hippocampal lesions in rat. Psychobiology, 15, 58-63.

OKaICHI, H., \& Oshima, Y. (1990). Choice behavior of hippocampectomized rats in the radial arm maze. Psychobiology, 18, 416-421.

O'KeEFE, J. (1991). The hippocampal cognitive map and navigational strategies. In J. Paillard (Ed.), Brain and space (pp. 273-295). Oxford: Oxford University Press.

O'KeEFE, J., \& CONWAY, D. H. (1980). On the trail of the hippocampal engram. Physiological Psychology, 8, 229-238.

O'KeEFE, J., \& NADEL, L. (1978). The hippocampus as a cognitive map. Oxford: Oxford University Press, Clarendon Press.

Packard, M. G., \& MCGaugh, J. L. (1992). Double dissociation of fornix and caudate nucleus lesions on acquisition of two water maze tasks: Further evidence for multiple memory systems. Behavioral Neuroscience, 106, 439-446.

Pico, R. M., Gerbrandt, L. K., Pondel, M., \& Ivy, G. (1985). During stepwise cue deletion, rat place behaviors correlate with place unit responses. Brain Research, 330, 369-372.
PouCET, B. (1993). Spatial cognitive maps in animals: New hypotheses on their structure and neural mechanisms. Psychological Review, 100, 163-182.

SaKamoto, T., \& OKaICHI, H. (1997). The effects of a fimbria-fornix lesion on distance discrimination in rats. Japanese Journal of Psychology, 68, 38-42.

SAVE, E., Poucet, B., Foreman, N., \& Buhot, M. (1992). Object exploration and reaction to spatial and nonspatial changes in hooded rats following damage to parietal cortex or hippocampal formation. $\mathrm{Be}$ havioral Neuroscience, 106, 447-456.

Whishaw, I., \& Mittelmam, G. (1986). Visits to stars, routes, and places by rats (Rattus norvegicus) in swimming pool navigation tasks. Journal of Comparative Psychology, 100, 422-431.

(Manuscript received July 22, 1997;

revision accepted for publication February 23, 1998.) 